\title{
Supraclavicular flap in head and neck reconstruction: experience in 50 consecutive patients
}

\author{
Kishore Sandu • Philippe Monnier • \\ Philippe Pasche
}

Received: 17 March 2011/Accepted: 23 August 2011/Published online: 23 September 2011

(C) Springer-Verlag 2011

\begin{abstract}
The supraclavicular flap (SCF) is a fasciocutaneous flap used to cover head, oral, and neck region defects after tumor resection. Its main vascular supply is the supraclavicular artery and accompanying veins and it can be harvested as a vascularised pedicled flap. The SCF serves as an excellent outer skin cover as well as a good inner mucosal lining after oral cavity and head-neck tumor resections. The flap has a wide arc of rotation and matches the skin colour and texture of the face and neck. Between March 2006 and March 2011, the pedicled supraclavicular flap was used for reconstruction in 50 consecutive patients after head and neck tumor resections and certain benign conditions in a tertiary university hospital setting. The flaps were tunnelized under the neck skin to cover the external cervicofacial defects or passed medial to the mandible to give an inner epithelial lining after the oral cavity and oropharyngeal tumor excision. Forty-four of the 50 patients had 100\% flap survival with excellent wound healing. All the flaps were harvested in less than $1 \mathrm{~h}$. There were four cases of distal tip desquamation and two patients had complete flap necrosis. Distal flap desquamation was observed in SCFs used for resurfacing the external skin defects after oral cavity tumor ablation and needed only conservative treatment measures. Total flap failure was encountered in two patients who had failed in previous chemoradiotherapy for squamous cell cancer of the floor of mouth and tonsil, respectively, and the SCF was used in mucosal defect closure after tumor ablation. The benefits of a pedicled fasciocutaneous supraclavicular flap are clear; it
\end{abstract}

K. Sandu $(\varangle) \cdot$ P. Monnier · P. Pasche

Department of Otorhinolaryngology,

University Hospital CHUV, Lausanne, Switzerland

e-mail: Kishore.Sandu@chuv.ch is thin, reliable, easy, and quick to harvest. In head, face and neck reconstructions, it is a good alternative to free fasciocutaneous flaps, regional pedicled myocutaneous flaps, and the deltopectoral flap.

Keywords Supraclavicular flap ·

Head neck reconstruction

\section{Introduction}

The goal of reconstructive surgical procedures after tumor ablation in the head and neck is not limited to cover the defect, but also to recreate anatomical functional units in three dimensions with similar skin colour and texture match as the original skin at the recepient site. Reconstructional surgeons have found that flaps designed around the shoulder are ideal for skin loss defects of the face and neck: the more adjacent the donor site the better skin match at the recepient site [1]. The supraclavicular flap (SCF) is a fasciocutaneous flap used extensively to resurface the neck and face in the patients after severe burn injuries [2]. It has the advantage of providing an optimum skin colour and texture match in the cervicofacial area. Over the past three decades, thanks to several anatomic studies conducted by various researchers, our knowledge of the skin vascular anatomy and physiology has led to several advancements in flap harvesting techniques. As a result, 'forgotten' flaps like the SCF has been rediscovered by several groups after an extended period of absence from the literature $[1-3,7]$. To our knowledge, this flap has rarely been described in otorhinolarygology literature for head and neck reconstruction after tumor ablation. It is a versatile flap adding to the armamentarium of the head and neck reconstructive surgeon. 


\section{Flap anatomy}

The supraclavicular artery is a perforator that arises from the transverse cervical artery in $93 \%$ and the suprascapular artery in $7 \%$ of the cases [1]. The artery ranges $1.0-1.5 \mathrm{~mm}$ in diameter, is constant in its location and offers $3-4 \mathrm{~cm}$ of vascular pedicle during harvesting of the flap. Pallua et al. $[1,2]$ have shown that in all the cases the artery is found in a triangle formed by the dorsal edge of the sternocleidomastoid muscle anteriorly, the external jugular vein posteriorly, and the medial part of the clavicle forming the base of this triangle. Same authors reveal that the artery exits $3 \mathrm{~cm}$ above the clavicle at a distance of approximately $8 \mathrm{~cm}$ from the sternoclavicular joint and is about $2 \mathrm{~cm}$ dorsal to the sternocleidomastoid muscle. Usually there are two veins draining the flap. One vein is found running adjacent to the artery and it drains into the transverse cervical vein; it has a mean diameter of $0.8-1.6 \mathrm{~mm}$. The second vein ranging $0.4-1.0 \mathrm{~mm}$ in diameter drains either into the external jugular vein or the subclavian vein [1].

\section{Angiosome of the supraclavicular artery}

In all the cases, the vascular territory extends from the supraclavicular region till the shoulder cap. The area of this angiosome ranges approximately $10 \mathrm{~cm}$ in width and $22 \mathrm{~cm}$ in length $[2,4]$.

\section{Materials and methods}

Between March 2006 and March 2011, the supraclavicular flap was used in 50 patients ( 38 males, 12 females) for complex face, head and neck reconstructions after tumor resection. The median age of the patients was 51 years (range 42-80 years) with a follow-up of 6-28 months. SCF was used either exclusively for an external skin or an internal oral mucosal defect closure or in combination with a composite flap reconstruction. Twenty-four patients had T3-4 squamous cell carcinoma SCC of the oral cavity (12 buccal mucosa, 2 retromolar trigone, 10 floor of mouth) and 2 were with T2 SCC of the tonsil. Three patients had carcinoma of the parotid gland (two adenoid cystic, one SCC) and needed excision of the overlying skin. Five patients were treated for T2-4 malignant melanoma of the cheek. One patient was referred for a ballistic injury to the lower face.

Seven patients had basal cell cancer involving the pinna, lateral face or the adjoining areas and one had involvement of the cheek. One patient had undergone a laryngotracheal diversion for severe intractable aspiration. He developed necrosis of the peritracheostomal skin which needed a flap cover. Our series consisted of three patients who developed a pharyngeal fistula (two patients following a total laryngectomy after the failed chemoradiation and the third patient who had undergone a diverticulectomy plus cricomyotomy for the treatment of Zenker's diverticulum). The fistulas in all the three cases measured less than $2 \mathrm{~cm}$ and had not responded to conservative treatment measures.

Seventeen cases were salvage surgeries after the failed primary chemoradiotherapy. Indications of using the SCF and the patient series are outlined in Table 1 . SCF was used exclusively as an outer (skin) cover in 42 cases. Oral and oropharyngeal mucosal defects were reconstructed using the SCF in five patients. In 6 patients, the flap was used in combination with an osseocutaneous (OC) flap (5 fibula, 1 iliac crest) and in 19 patients it was used along with a pedicled myocutaneous (MC) flap (6 latissimus dorsi-LD, 12 pectoralis major-PMMC). When used in combination with the $\mathrm{OC}$ and MC flaps, the skin paddle of the composite flap was used for the oral mucosal defect closure and the SCF was used to line the external skin defect.

Average ablative skin defects at the recepient site measured approximately $7 \times 15 \mathrm{~cm}$ and the average mucosal defects were approximately $4 \times 5 \mathrm{~cm}$. The average SCF dimensions were approximately $6 \times 17 \mathrm{~cm}$. In four cases, the flap was autonomised 10 days prior to the reconstruction of a high facial defect (three patients) and oropharyngeal reconstruction (one patient), who then had a two-stage reconstruction. Autonomisation provided an additional flap length of 3-4 cm. All other defects involving the lateral face, cheek, chin, oral cavity (including the

Table 1 Surgical indications for the supraclavicular flap

\begin{tabular}{ll}
\hline Indications & $\begin{array}{l}\text { Number of } \\
\text { patients } \\
(n=50)\end{array}$ \\
\hline $\begin{array}{l}\text { Outer skin closure }(n=42) \\
\text { In combination with a free osseocutaneous flap }\end{array}$ & 6 \\
$\quad$ 5 fibula, 1 iliac crest) & 19 \\
In combination with a pedicled myocutaneus flap & \\
(6 latissimus dorsi, 13 pectoralis major) & 5 \\
Malignant melanoma & 3 \\
Parotid tumor & 8 \\
Basal cell cancer & 1 \\
Peritracheostomal skin loss & \\
Mucosal closure $(n=5)$ & 3 \\
Oral cancer (T3-4 SCC of floor of mouth) & 2 \\
Oropharyngeal cancer (T2 SCC tonsil) & \\
Pharyngeal fistula closure $(n=3)$ & 2 \\
Total laryngectomy & 1 \\
Zenker's diverticulectomy &
\end{tabular}


second patient needing an oropharyngeal mucosal reconstruction), and the paranasal area were reconstructed primarily.

\section{Flap harvesting}

A preoperative angiography is performed so as to assess the presence and integrity of the supraclavicular artery. Intraoperatively, the artery is marked using a Doppler probe in the lower aspect of the neck in the supraclavicular fossa. The flap pedicle is found in a triangle (Fig. 1) bound by the posterior edge of the sternocleidomastoid muscle medially, the external jugular vein laterally, and the medial edge of the clavicle inferiorly.

The pedicle and the required dimensions of the flap skin paddle are marked over the shoulder. After infiltrating with 1:2,00,000 lidocaine-adrenaline solution, the incision begins above the clavicle and follows around the shoulder. The skin, subcutaneous tissue, and the fascia over the deltoid muscle are incised. The fascia is fixed with the skin using 4.0 vicryl suture. Further dissection is continued in the subfascial plane over the deltoid muscle and the flap is raised in the distal to proximal (or lateral to medial) direction. This distal to proximal flap harvest is safer than

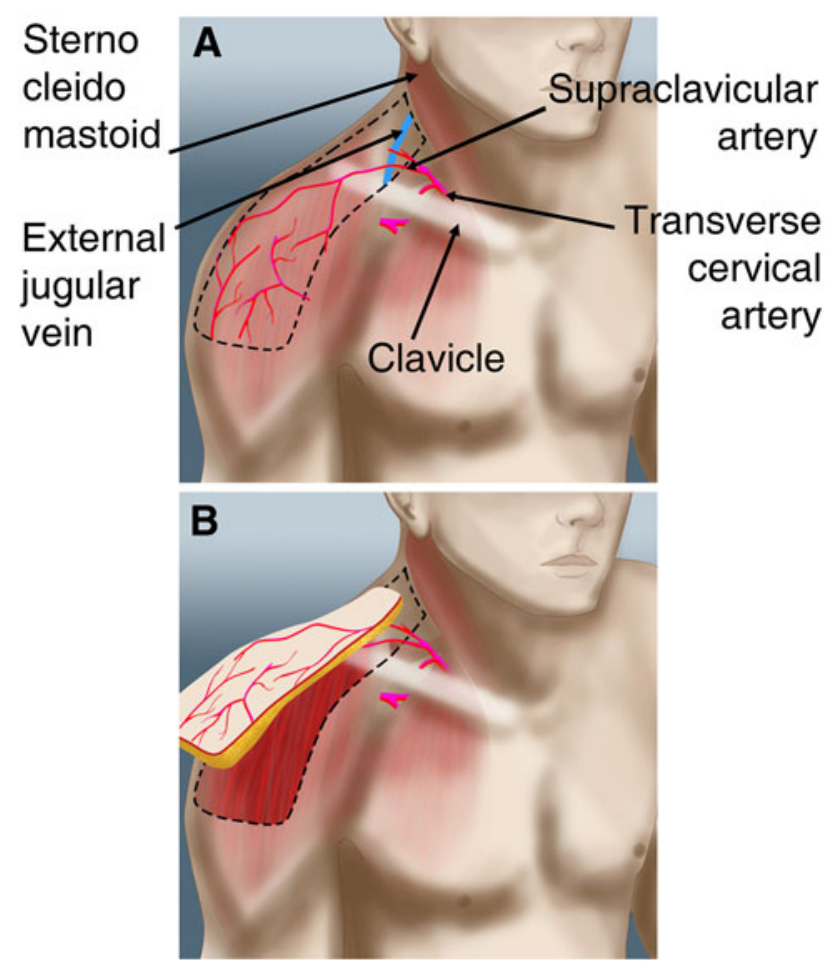

Fig. 1 The supraclavicular artery is found in a triangle formed by the dorsal edge of the sternocleidomastoid muscle medially, the external jugular vein laterally, and the medial part of the clavicle inferiorly an anterior to posterior harvest direction which could inadvertently injure the flap pedicle. In the medial third of the flap, the pedicle can be identified by transillumination and confirmed by the Doppler. Depending on the flap dimensions required, the medial incision is taken to cut only the skin and subcutaneous tissue, avoiding damage to the pedicle. The XI cranial nerve is identified and preserved till its entry into the trapezius.

The next step is to tunnelize the flap under the neck skin, remaining lateral to the sternocleidomastoid muscle (SCM). Mobilisation of the flap to get it medial to the SCM could kink the vascular pedicle and hence should be avoided. It is important to note that the preparation of the flap is carried out at the subfascial level in its distal part (over the deltoid region), then beginning to tunnelise the proximal portion of the flap at the subcutaneous level. Dissection can be continued to the pivot point of the supraclavicular artery at its origin from the transverse cervical artery. Once in the vicinity of the pedicle, dissection is continued with a bipolar coagulation forceps to avoid injury to the pedicle. The distal portion of the flap is trimmed until healthy bleeding is noted. Finally, the fasciocutaneous flap is pulled through the neck tunnel and placed in the defect. Use of the SCF to close external skin defects is illustrated in Figs. 2 and 3. For both oral and oropharyngeal defects, the flap is passed medial to the mandible to avoid compression of the vascular pedicle. The donor site is closed primarily by extensive subcutaneous undermining and approximation of wound edges. Larger defects may require skin grafting.

Cervical, chin, paranasal, cheek, and lower face defects were closed by the SCF harvested in single-stage. For higher facial, temporal, and oropharyngeal defects the SCF is autonomized 7-10 days prior to the actual surgical intervention. In such a two-stage procedure performed under local anesthesia and sedation, the incision passes 3-4 $\mathrm{cm}$ distal to the deltoid prominence on the flexor aspect of the arm to gain an additional flap length. The flap is dissected in the subfascial plane to identify the vascular pedicle as described earlier. Extensive flap mobilisation is avoided and XI cranial nerve not identified. A silastic sheet $0.005 \mathrm{~cm}$ measuring $1 \mathrm{~cm}$ smaller than the paddle dimension is placed over the deltoid muscle. The silastic sheet avoids refixation of the flap with the underlying tissue and muscle, while permitting optimal vascularisation of the additional skin length solely by the fasciocutaneous perforators. The skin is resutured with continuous 3.0 prolene suture leaving in a penrose drain. During definitive surgery, the silastic sheet is removed and sufficient additional length is gained by the randomised autonomisation.

In the three patients who had pharyngeal salivary fistula, the SCF was harvested as described earlier and the skin paddle was deepithelialised. The margins of the fistulous 
Fig. 2 A 42-year-old male patient was diagnosed to have T2N2bM0 SCC of the right retromolar trigone. $\mathrm{He}$ underwent a marginal mandibulectomy followed by radiochemotherapy. In 2 years, there was local recurrence with skin infiltration (a, b). He then underwent a segmental mandibular resection including external skin excision (c). Mandibular reconstruction consisted of a free fibular graft $(11 \mathrm{~cm})$ using the skin for internal mucosal closure (d). External skin defect measuring $8 \times 12 \mathrm{~cm}$ was repaired with a pediculated supraclavicular flap (e). f the patient 6 months postoperatively

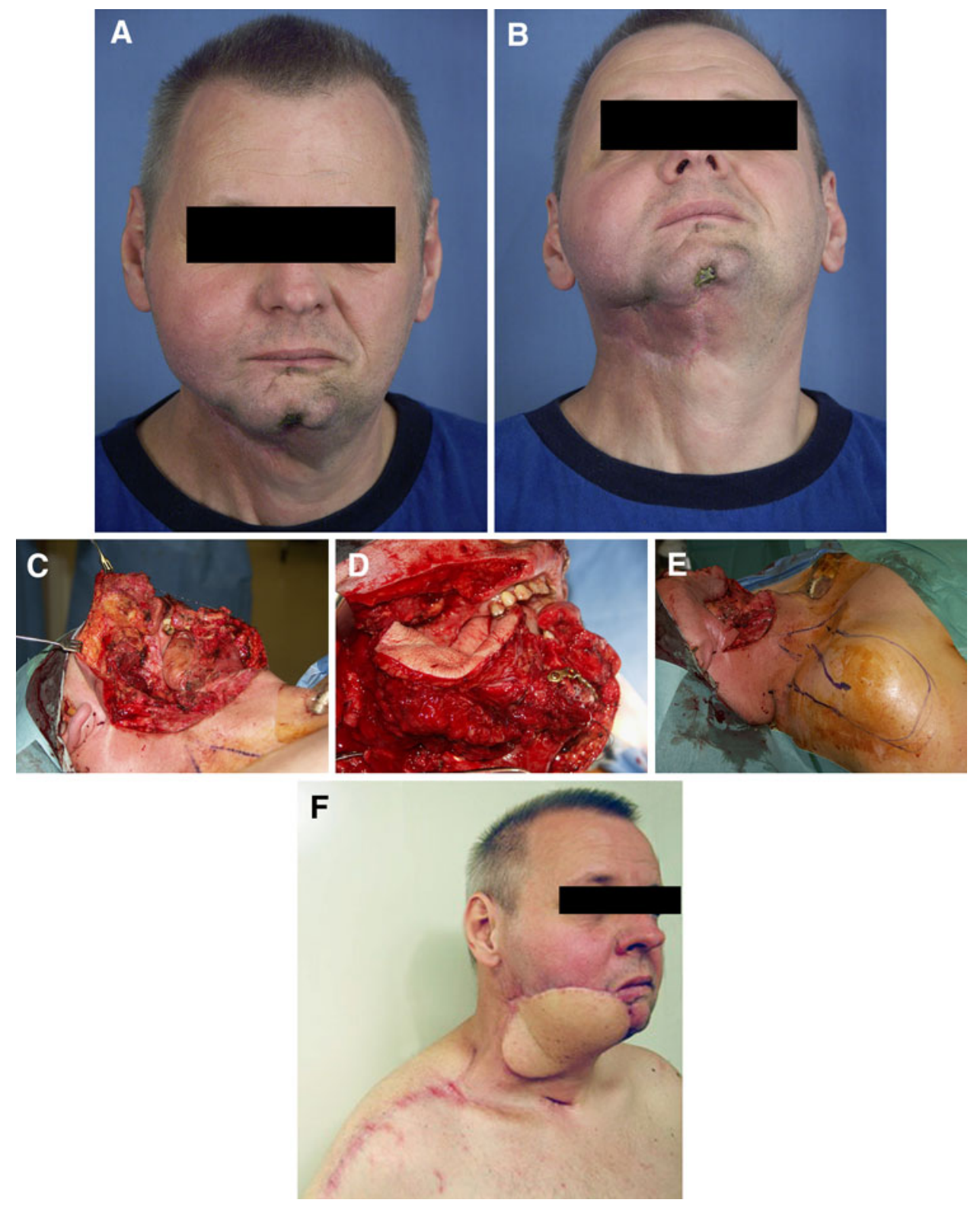

opening were freshened and mobilised so as to achieve a primary closure. The SCF was then used further to reinforce and vascularize the repair. Postoperative surveillance of the flap used in external skin or oral defect closure was done every $2-4 \mathrm{~h}$ in the first $72 \mathrm{~h}$ assessing the skin paddle temperature and its recoloration. Buried flaps in the cases of pharyngeal fistulas were not monitored.

\section{Results}

Forty-four patients (88\%) had a satisfactory flap healing and complete integration into the resection site. Distal flap desquamation occurred in four cases $(8 \%)$ which healed completely by secondary intention requiring no further surgical correction. Two patients (4\%) had total necrosis and required a free radial forearm flap. The SCF was used at salvage surgery in these two patients for mucosal defect closure after the failed primary chemoradiotherapy for floor of mouth and tonsillar tumor, respectively. We attributed the flap failure to a radiated recepient bed and probably an inadequate oral and oropharyngo-cervical tunnel through which the SCF was passed for mucosal defect closure. Other three patients in whom the SCF was used to close the mucosal defect following a primary surgical tumor ablation showed an excellent healing without complications. Postoperative barium swallow study in the three patients with pharyngeal fistula was satisfactory and they all started oral feeds at the end of the second postoperative week; requiring no other surgical intervention. 

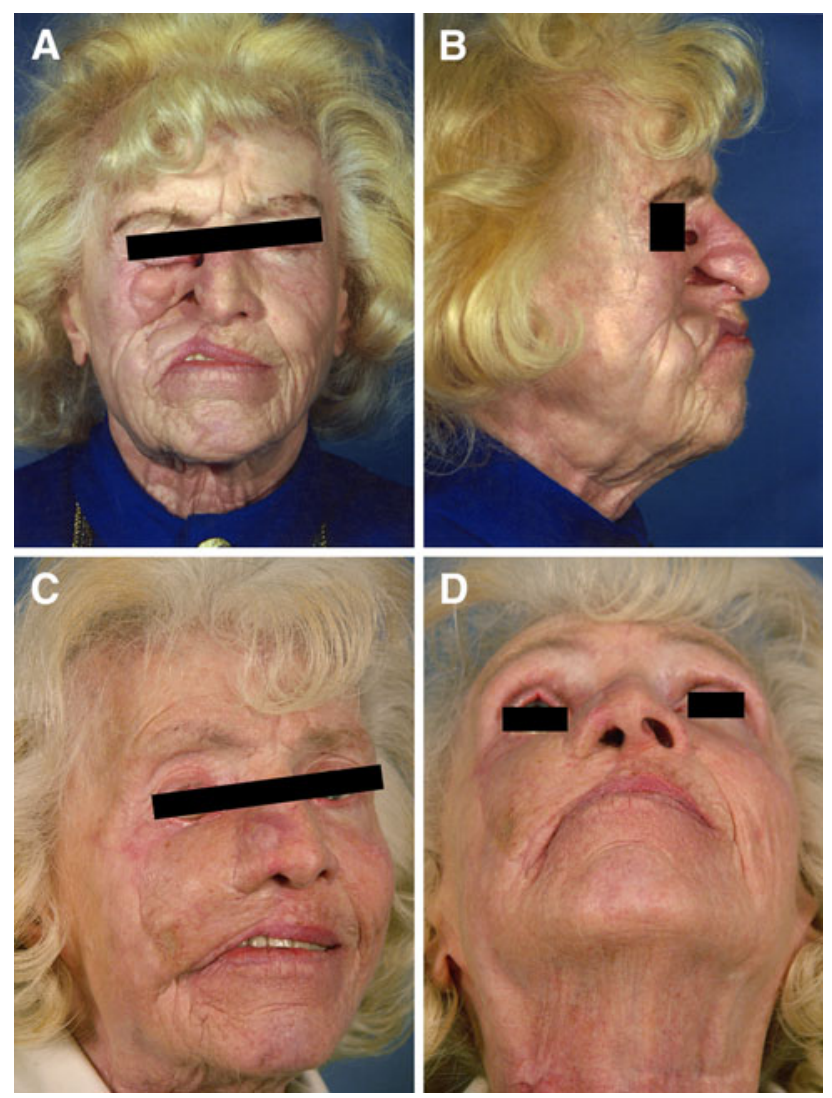

Fig. 3 This 80-year-old female patient underwent maxillectomy and resection of the orbital floor for an invasive basal cell carcinoma of the cheek (a, b). Her midfacial defect measuring $5 \times 6 \mathrm{~cm}$ was reconstructed with a supraclavicular flap. (c, d) the patient 1 year postoperatively

Donor site defects were encountered in six patients (12\%) which received VAC (vacuum assisted closure) followed by skin grafting. Eight patients complained of mild to moderate pain referred to the shoulder joint, which responded well to NSAID's and physiotherapy. Interestingly, two-third of the patients complained of non-irritating referred sensation to the shoulder on touching the flap. Similarly, the patients in whom the SCF was used to treat a pharyngeal fistula, noted this referred sensation on deglutition and when liquid or food passed the fistulous site.

\section{Discussion}

SCF has been used extensively by plastic surgeons for the reconstruction of neck and face after severe burn sequellae. The supraclavicular area and the shoulder are easy to conceal and flaps designed in this area are advantageous in reconstruction procedures of the face and neck. Flaps from the shoulder were introduced by Mutter [1] as early as 1842 and several modifications have been presented in subsequent years. In 1979, Lamberty [4] was the first to describe the axial pattern of the shoulder flap based on the supraclavicular artery. In 1996, Pallua [1, 2] identified the vascular pedicle of the SCF, described its angiosomes and extended the versatility of the flap to head and neck reconstruction.

Soft-tissue oncologic resections of the head, face and neck often results in complex reconstructive problems that require a dependable local, regional or free flap to restore both the form and function. In all these reconstructions, significant donor site morbidity should be avoided. Thin pliable flap tissue with good colour and texture match are ideal to cover the external skin defects of the neck and face. Local flaps would be well suited in such a scenario, but their use may be restricted because of previous radiotherapy or surgery. Regional myocutaneous flaps (pectoralis major-PMMC and latissimus dorsi-LD) are bulky and often cannot be used in pure oropharyngeal reconstructions. They usually require a condyle non-sparing or segmental mandibulectomy to accomodate them. Even after marginal mandibulectomy, these flaps are too voluminous to be adapted lateral to the remaining mandibular bone. Insetting these flaps medial to the mandible is difficult and has the risk of pedicle compression against the mandible [6]. The regional myocutaneous flaps need extensive muscle dissection leading to increased donor site morbidity and complications like hematomas. Placing the patient in a lateral decubitus position during harvest of the latissimus dorsi or trapezius flaps and then rechanging the position for flap accomodation into the defect can be quite cumbersome and increases the surgical time. Also, flaps from the trunk do not provide homogeneity of texture, colour and thickness to the neck and face region. The superior trapezius flap has a limited arc of rotation and cannot be used for closure of defects that cross the midline of the face and neck anteriorly [5, 6]. Platysma flap could be ideal in these reconstructions, but the need to preserve the facial artery and vein may compromise the clearance of level I neck nodes which is not desirable in oral cavity tumors. Microsurgical transplantation of free flaps (radial forearm, anterolateral thigh, and parascapular) provides pliable thin vascularized tissue to fill the large defects, though they lack colour match to the face and neck. In addition, these flaps need personnel trained in microsurgical techniques and special postoperative monitoring. High risk patients (those with advanced age, advanced tumors, poor nutrition or multiple medical issues) are not good surgical candidates for potentially prolonged microsurgery, therefore, regional flaps remain the preferred technique in these more difficult cases. The SCF is an ideal flap suited for these patients. It is thin with the best skin colour and texture match and can be harvested in less than $1 \mathrm{~h}$.

Oncological reconstructive teams need to have various options of flap reconstructions in their armamentarium. 
Sometimes, we face situations wherein the Allen's test is negative bilaterally, meaning, a free radial forearm flap, the present workhorse of reconstructive surgery would be difficult to use. A SCF could be a possible alternative in such cases. We have used this flap in combination with free osseocutaneous or pedicled myocutaneous flaps in cases with large oromandibulo-cervical defects with skin ablation: the outer skin defect being reconstructed by the SCF and the inner mucosal defect by the skin paddle of these composite flaps. Unlike the DP flap, the SCF is harvested and adapted definitively at the recepient site in a single stage. Therefore, it has progressively replaced the DP flap for face and neck reconstructions at our institution.

We found following advantages with the SCF:

1. it is easy and quick to harvest

2. it has an excellent skin colour and tissue texture matching the face and neck

3. it has a consistent and wide arc of rotation with a long pedicle which is well suited for oral, oropharyngeal, and high facial defects

4. it has simple postoperative surveillance as against a free tissue transfer

5. there is very little donor site morbidity. A shoulder defect requiring a skin graft can be well hidden

6. it is a good alternative, when Allen's test (for free radial forearm flap) is negative bilaterally, or in difficult recepient neck vessels

7. it has a single-stage flap closure as against the Bakamjian DP flap needing an interval division. During this interval period (about 3 weeks), the DP flap is cumbersome to maintain and may not be cosmetically acceptable to the patient.

Contra-indications for the SCF are:

1. radical or functional neck dissection requiring ligation of the vascular pedicle

2. doubtful pedicle integrity on preoperative angiogram

During harvest of the SCF, if a long flap length is not needed, a cuff of soft-tissue is left around the origin of the artery from the transverse cervical artery which avoids inadvertent damage to the vascular pedicle. A distal to proximal harvest is more safer for the pedicle than an anterior to posterior harvest. Care should be taken when performing the tunnel for flap adaptation. An inadequate aperture can lead to pedicle compression. Tunneling of the SCF under radiated tissue or in certain cases of previous scarring can be difficult. The scar band may impair the blood flow to the distal flap where the transferred tissue is most needed. In such cases, it is better to connect the proximal skin island flap to the neck dissection incision. Chiu et al. [7] routinely perform this technique without tunneling and have not observed any skin necrosis at the trifurcation site.
Recently, in a large series of 103 supraclavicular flaps reported by Vinh et al. [8] used exclusively in treating neck contractures, 94\% flaps survived completely, $4 \%$ exhibited superficial distal necrosis, and $2 \%$ patients had total necrosis. Chiu et al. [7] reported using the supraclavicular island flap in 18 patients requiring reconstruction of head and neck oncologic defects. The same authors had one patient (5\%) with complete flap necrosis and four with minor recepient site complications which needed only local conservative treatment. In our 50 patients, we noted minor distal flap desquamation in 4 cases $(8 \%)$ which responded well to conservative measures. We had two cases $(4 \%)$ of total flap necrosis in patients who had failed primary chemoradiotherapy for floor of mouth and tonsillar SCC, respectively. In both these patients, the flap was used for mucosal defect closure after tumor ablation and was tunneled under a radiated skin. This could have been compressive over the pedicle inspite of an adequate orocervical aperture and tension free flap delivery at the recepient site. This pedicle compression along with a radiated recepient site caused the total flap necrosis. In such cases, we recommend joining the proximal flap incision with the neck dissection incision to maximise the flap microcirculation.

In our series, the average SCF dimensions were approximately $6 \times 17 \mathrm{~cm}$, though flaps up to $11 \times 21 \mathrm{~cm}$ have been harvested with good results [8]. Even bilateral SCF's have been used successfully in large face and neck defects $[3,7,8]$. When SCF is used for oropharyngeal or high facial defect reconstruction, a longer flap extending beyond the deltoid muscle on the flexor surface of the arm is needed. In such a situation, we advocate an autonomisation of the flap under local anesthesia 7-10 days prior to the actual surgical intervention. This provides an addtional length of 3-4 cm. In oropharyngeal reconstructions, especially when the mandible is spared, a SCF is better adapted than pedicled myocutaneous flaps (PMMC, latissimus dorsi) which are bulky and have a greater risk of pedicle compression against the mandible. In such cases, the SCF passed medial to the mandible provides excellent flap tissue to close these mucosal defects. Presently, our experience using the SCF for oral/oropharyngeal mucosal defect closure is limited only to five patients and a larger series is certainly required for better acceptance of the flap in these cases. In our three patients with pharyngeal fistulas, the SCF was buried under the neck skin and used to increase the vascularity and reinforce the closure of the fistulous site. Monitoring of such a flap is not done as it has both an artery and a vein originating from its parent vessels [7]. The referred sensation to the shoulder on tactile stimulation of the flap was experienced by majority of our patients. This sensation is not painful or disturbing, but warrants further research to know its exact mechanism. 


\section{Conclusions}

The pedicled supraclavicular faciocutaneous flap (SCF) provides thin pliable tissue with an excellent skin colour and texture match making it ideal to resurface external skin losses in face and neck reconstructions. Because of several advantages, the SCF should certainly be prefered to the deltopectoral flap. In certain selected patients, it can be an alternative to free fasciocutaneous flaps for mucosal defect reconstruction in oral and oropharyngeal tumors. The SCF in combination with free osseocutaneous or pedicled myocutaneous flaps is well suited for complex orocervical defects.

Conflict of interest There are no financial aids or conflicts of interests in the preparation of this article.

\section{References}

1. Pallua N, Noah EM (2000) The tunneled supraclavicular island flap: an optimized technique for head and neck reconstruction. Plast Reconstr Surg 105(3):842-851
2. Pallua N, Machens HG, Rennekampff O, Becker M, Berger A (1997) The fasciocutaneous supraclavicular artery island flap for releasing postburn mentosternal contractures. Plast Reconstr Surg 99(7):1878-1884

3. Vinh VQ, Ogawa R, Anh TV, Hyakusoku H (2007) Reconstruction of neck scar contractures using supraclavicular flaps: retrospective study of 30 cases. Plast Reconstr Surg 119(1):130-135

4. Lamberty BG (1979) The supra-clavicular axial patterned flap. Br J Plast Surg 32(3):207-212

5. Margulis A, Agam K, Icekson M, Dotan L, Yanko-Arzi R, Neuman R (2007) The expanded supraclavicular flap, prefabricated with thoracoacromial vessels for reconstruction of postburn anterior cervical contractures. Plast Reconstr Surg 119(7):20722077

6. Urken ML (1995) Trapezius system. In: Urken ML et al (eds) Atlas of regional and free flaps for head and neck reconstruction. Raven Press, New York, pp 29-48

7. Chiu ES, Liu PH, Friedlander PL (2009) Supraclavicular artery island flap for head and neck oncologic reconstruction: indications, complications and outcome. Plast Reconstr Surg 124(1):115-123

8. Vinh VQ, Anh TV, Ogawa R, Hyakusoku H (2009) Anatomical and clinical studies of the supraclavicular flap: analysis of 103 flaps used to reconstruct neck scar contractures. Plast Reconstr Surg 123(5):1471-1480 